\title{
Clinical evaluation of the failure rates of metallic brackets
}

\author{
Fábio Lourenço ROMANO'1, Américo Bortolazzo CORRER ${ }^{2}$, Lourenço CORRER-SOBRINHO ${ }^{3}$, Maria Beatriz Borges \\ de Araújo MAGNANI ${ }^{4}$, Antônio Carlos de Oliveira RUELLAS ${ }^{5}$ \\ 1- PhD, Professor, Department of Pediatric Clinic, Preventive and Community Dentistry, School of Dentistry of Ribeirão Preto, University of São Paulo, Ribeirão \\ Preto, SP, Brazil. \\ 2- PhD, Department of Restorative Dentistry, Piracicaba Dental School, University of Campinas, Piracicaba, SP, Brazil. \\ 3- PhD, Full Professor, Department of Restorative Dentistry, Piracicaba Dental School, University of Campinas, Piracicaba, SP, Brazil. \\ 4- PhD, Professor, Department of Pediatric Dentistry, Piracicaba Dental School, University of Campinas, Piracicaba, SP, Brazil. \\ 5- PhD, Professor, Department Pediatric Dentistry and Orthodontics, Dental School, Federal University of Rio de Janeiro, Rio de Janeiro, RJ, Brazil.
}

Corresponding address: Prof. Dr. Fábio Lourenço Romano - Faculdade de Odontologia de Ribeirão Preto - USP - Departamento de Clínica Infantil, Odontologia Preventiva e Social - Av. do Café, s/n - Monte Alegre - Ribeirão Preto - SP - Brasil - 14040-904 - e-mail: fabioromano@forp.usp.br

Received: June 27, 2010 - Modification: July 31, 2011 - Accepted: September 05, 2011

\section{ABSTRACT}

$\mathrm{O}$ bjectives: The aim of this study was to evaluate in vivo the bonding of metallic orthodontic brackets with different adhesive systems. Material and Methods: Twenty patients (10.5-15.1 years old) who had sought corrective orthodontic treatment at a University Orthodontic Clinic were evaluated. Brackets were bonded from the right second premolar to the left second premolar in the upper and lower arches using: Orthodontic Concise, conventional Transbond XT, Transbond XT without primer, and Transbond XT associated with Transbond Plus Self-etching Primer (TPSEP). The 4 adhesive systems were used in all patients using a split-mouth design; each adhesive system was used in one quadrant of each dental arch, so that each group of 5 patients received the same bonding sequence. Initial archwires were inserted 1 week after bracket bonding. The number of bracket failures for each adhesive system was quantified over a 6-month period. Results: The number of debonded brackets was: 8- Orthodontic Concise, 2- conventional Transbond XT, 9- Transbond XT without primer, and 1- Transbond XT + TPSEP. By using the KaplanMeier methods, statistically significant differences were found between the materials $(p=0.0198)$, and the Logrank test identified these differences. Conventional Transbond XT and Transbond XT + TPSEP adhesive systems were statistically superior to Orthodontic Concise and Transbond XT without primer $(p<0.05)$. There was no statistically significant difference between the dental arches (upper and lower), between the dental arch sides (right and left), and among the quadrants. Conclusions: The largest number of bracket failures occurred with Orthodontic Concise and Transbond XT without primer systems and few bracket failures occurred with conventional Transbond XT and Transbond XT+TPSEP. More bracket failures were observed in the posterior region compared with the anterior region.

Key words: Composite resins. Orthodontic brackets. Orthodontics.

\section{INTRODUCTION}

Many commercially available orthodontic bonding materials have been experimentally evaluated in laboratories ${ }^{13,27,29}$, but not all were clinically tested to confirm their efficiency and effectiveness. Despite the lack of clinical evaluation, these materials are commonly used by orthodontists for bonding orthodontic appliances.
Amongst the most widely used materials for bonding orthodontic accessories directly to dental enamel, composite resins stand out. However, in spite of offering adequate adhesion, these materials also require a moisture-free operative field and a step-by-step clinical technique ${ }^{3,5}$. For bonding brackets using conventional composites, the enamel surface must be adequately prepared according to the following steps: prophylaxis with pumice/water 
slurry, washing and drying, 37\% phosphoric acid etching for 15-30 s washing and drying, and finally, application of the bonding agent and placement of the composite resin.

Orthodontic Concise (3M Brazil, Sumaré, SP, Brazil) and Transbond XT (3M Unitek, Monrovia, CA, USA) composites, when used according to the manufacturer's recommendations, require use of their primers (respectively, fluid resins A and $B$, and $\mathrm{XT}$ primer). There is controversy about the use of primers, since some authors did not find differences in the adhesion of orthodontic accessories to enamel with or without previous priming of enamel surface $^{16,20}$. This material moistens and penetrates the enamel and protects the etched tooth surface that will not suffer decalcification caused by plaque and food residues ${ }^{20}$. Despite some advantages, eliminating the priming step would decrease chair time, keep the working field dry, and possibly reduce the bonding failure caused by contamination or moisture.

The self-etching primers (SEPs), which combine acid and primer in a single solution, have been recently developed $22,25,28$. SEPs are easy to be handled and can be applied even to contaminated surfaces, thus providing comfort for patient and short-time visits ${ }^{27,28}$. The Transbond Plus SelfEtching Primer (TPSEP; 3M Unitek) was tested in several laboratory 3,9,13,29 and clinical ${ }^{22,25,26}$ studies, with good adhesive results. Some studies have quantified the number of debonded brackets using TPSEP as an enamel conditioning agent before using Transbond XT for the bonding procedure ${ }^{10,17,24}$, while others have compared different self-etching agents and primers $2,7,22,23,25$. Nevertheless, no study has assessed four different adhesive systems in the same patient by using quadrant variations.

The purpose of the present study was to perform a 6-month clinical assessment of the failure rate of brackets bonded with Orthodontic Concise, conventional Transbond XT, Transbond XT without primer, and Transbond XT+TPSEP. The number of bracket failures was also compared between dental arches, regions, sides, and quadrants.

\section{MATERIAL AND METHODS}

This research protocol was approved by the Ethics Committee of Piracicaba Dental School, University of Campinas (UNICAMP), under protocol number $116 / 2008$.

Forty-two eligible patients were recruited from the waiting list of the Orthodontic Clinic at the Piracicaba Dental School for an initial screening, resulting in a final sample to 20 patients (13 females and 7 males) aged 10.5 to 15.1 years who met the inclusion criteria. Only individuals needing corrective orthodontic treatment but who had never been submitted to any type of orthodontic therapy were included in the study. The exclusion criteria were need for ortho-surgical treatment or tooth extractions for correction of malocclusion, presence of gold or ceramic dental crowns, presence of amalgam or composite restorations, congenital enamel defects, missing teeth, and craniofacial anomalies. Sex, age, race and malocclusion differences were ignored. The details of sample size, malocclusion types, number of brackets bonded with each adhesive system and patient distribution by sex and age are presented in Table 1.

The selected patients received corrective orthodontic treatment with fixed appliance placed in the upper and lower arches using the Edgewise technique. A total of 400 orthodontic brackets (Morelli, Sorocaba, SP, Brazil), 20 per patient, were bonded to the upper teeth $15,14,13,12,11,21$, $22,23,24$ and 25 and to the lower teeth 35, 34, $33,32,31,41,42,43,44$, and 45. Orthodontic bands were attached with glass-ionomer cement (Ketac-Cem, 3M ESPE, St. Paul, MN, USA) to the first and second molars.

Prior to the bracket bonding procedures, lip and cheek retractors were placed and all buccal surfaces were cleaned with a rubber cup and fluoride-free pumice stones (S.S. White, Petropolis, RJ, Brazil) and water for $10 \mathrm{~s}$ in each tooth at low speed; washing and drying took the same time. Next, cotton rolls were used to isolate and keep the operative field dry. Figure 1 lists the four adhesive systems used to bond the brackets.

With regard to Orthodontic Concise composite (3M Brazil), conventional Transbond XT composite (3M Unitek), and Transbond XT without primer, the dental enamel was previously etched with $37 \%$ phosphoric acid (Dentsply Dentsply Ind. e Com. Ltda., Petropolis, RJ, Brazil) for $15 \mathrm{~s}$, followed by washing and drying for the same time. However, TPSEP (3M Unitek) was used for conditioning the enamel surface prior to Transbond XT composite according to the manufacturer's recommendations, that is, the material was rubbed onto the enamel surface for three $s$ and then air jet was gently applied.

With regard to the bonding procedure, the brackets with a composite increment on their base were pressed against the enamel surface held with a pair of nippers (Orthopli Corp, Philadelphia, PA, USA), positioned and excess material was removed with a small scaler (Duflex, Juiz de Fora, MG, Brazil).

Brackets bonded with Transbond XT were light cured for $40 \mathrm{~s}$ (10 s for each face: mesial, distal, incisal or occlusal, and gingival) by using a halogen light device (XL 2500; 3M ESPE) with irradiance of $500 \mathrm{~mW} / \mathrm{cm}^{2}$ at a distance of $1 \mathrm{~mm}$ from the bracket. The light intensity was regularly gauged with a curing radiometer (Demetron, Danbury, CT, 
USA).

The adhesive systems were allocated by a split-mouth design: the mouth of each patient was divided into four quadrants with each system being used in one quadrant of the dental arch according to bonding sequence. Each bonding sequence was repeated in every 5 patients to assure an equal distribution among the quadrants and reduce bias because of patient chewing or parafunctional preferences (bonding sequence of 1 to 4 , Figure 2).

The brackets were bonded in all patients by a single operator in order to eliminate variation during a single visit. Immediately after the bonding procedures, the patients were instructed about the maintenance care of their appliances, oral hygiene, and type of diet to be avoided, such as hard foods, not to damage the orthodontic accessories. The lower arch brackets were bonded in such a way to avoid premature contact. Flexible nickel-titanium 0.012 " archwires were tied to the brackets with individual elastics 1 week after bonding. This care was taken to avoid that the initial pressure of the arch inside the bracket slot in the uneven teeth would promote premature debonding of the accessory, thus interfering in bond strength assessment. Later, rounded stainless

Table 1- Sample characteristics

\begin{tabular}{lcc}
\hline & Number & $\%$ \\
\hline Number of patients & 20 & \\
\hline Distribution of patients by gender & 7 & 35 \\
Male & 13 & 65 \\
\hline Female & 3 & 15 \\
\hline Distribution by age & 8 & 40 \\
\hline$<11$ years & 6 & 30 \\
\hline $11 / 12$ years & 1 & 5 \\
$12 / 13$ years & 1 & 5 \\
\hline $13 / 14$ years & 1 & 5 \\
14/15 years & 400 & \\
\hline$>15$ years & & 25 \\
Number of brackets & 100 & 25 \\
\hline Distribution of brackets by adhesive system & 100 & 25 \\
Orthodontic Concise & 100 & 25 \\
\hline Conventional Transbond XT & 100 & \\
\hline Transbond XT without primer & & 40 \\
\hline Transbond XT + TPSEP & 8 & 55 \\
\hline Distribution of patients by malocclusion & 11 & 5 \\
\hline Class I & 1 & \\
\hline Class II & & \\
\hline Class III & & \\
\hline
\end{tabular}

\begin{tabular}{|c|c|}
\hline Adhesive systems & Bonding procedures \\
\hline Orthodontic Concise & $\begin{array}{l}\text { Acid etching, mixing, and application of fluid resins ( } A \text { and } B \text { ), gentle air jet, } \\
\text { manipulation of pastes A and B of Orthodontic Concise composite, application on the } \\
\text { bracket base for bonding. }\end{array}$ \\
\hline Conv & $\begin{array}{c}\text { Acid etching, application of XT primer, gentle air jet, application of Transbond XT } \\
\text { composite on the bracket base for bonding. }\end{array}$ \\
\hline Transbond XT withou & Acid etching, application of Transbond XT composite on the bracket base for bonding. \\
\hline Transbond XT with primer-acid & $\begin{array}{l}\text { Application of TPSEP*, application of Transbond XT composite on the bracket base } \\
\text { for bonding. }\end{array}$ \\
\hline
\end{tabular}

*TPSEP=Transbond Plus Self Etching Primer

Figure 1-Adhesive systems and bonding procedures 


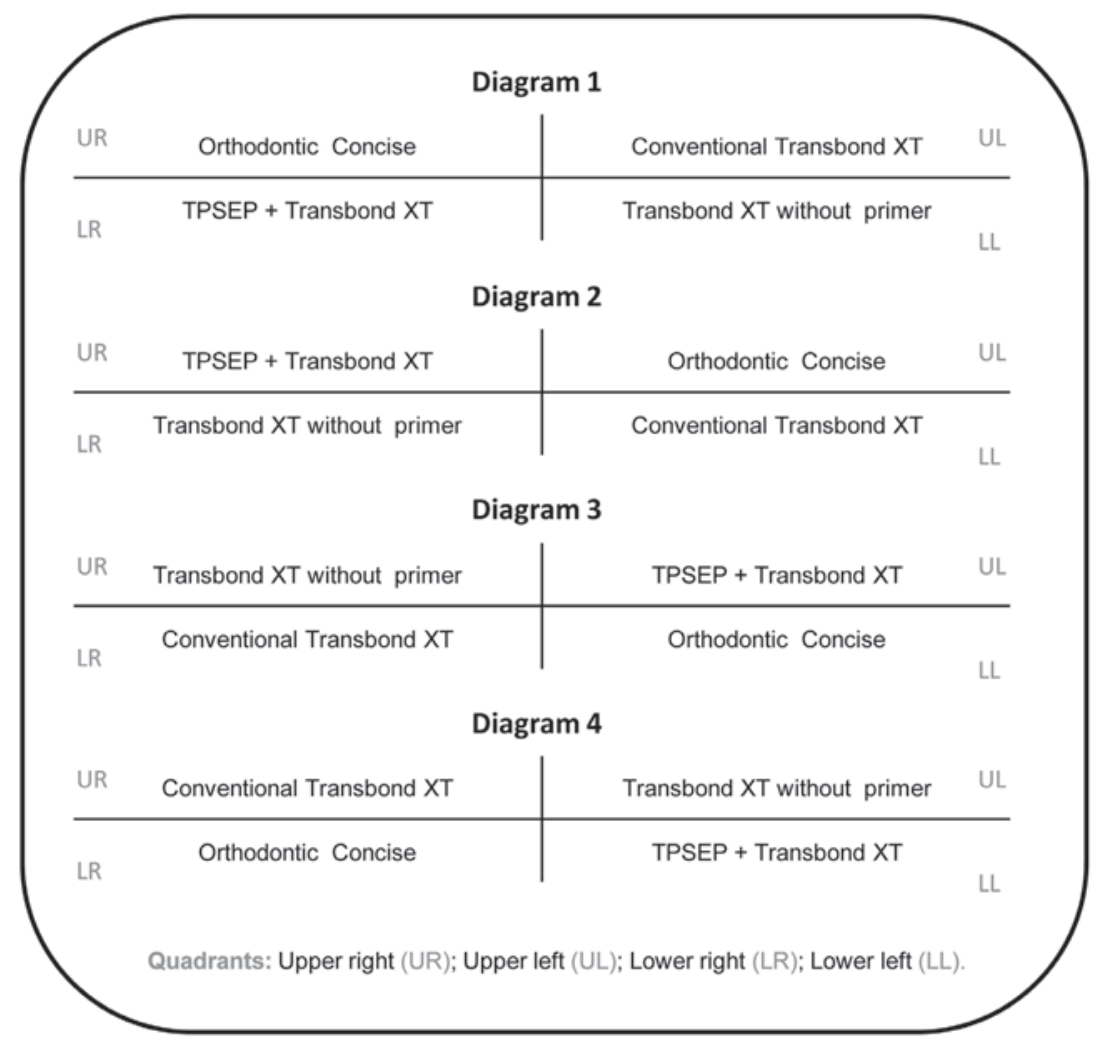

Figure 2- Distribution of adhesive systems in the four quadrants

steel archwires (in the sequence 0.016", 0.018" and $0.020^{\prime \prime}$ ) and then rectangular archwires (in the sequence $0.018 " x 0.025^{\prime \prime}$ and $0.019 " \times 0.025^{\prime \prime}$ ) were placed. The archwires were replaced every 3 weeks on average, the rectangular archwires being placed close to the end of the evaluation period, that is, approximately 6 months after the beginning of the treatment. The patients were seen every 21 days for checking bonding failures and for evaluating the corrective orthodontic treatment. During the 6-month evaluation period, any bond failure was recorded on a data collection sheet on the day the patient attended with the breakage. The first bond failure for each tooth was recorded by date and tooth number. Any failure was regarded as an "all-or-none" occurrence; subsequent bond failures in the same tooth were noted down, but were not included in the study. In the same appointment that the failure was perceived, the failed bracket was replaced using the same adhesive and bonding technique. The patients were maintained under treatment according to previously outlined orthodontic treatment plan. Treatment duration was established individually for each patient based on the malocclusion characteristics involved.

\section{Statistical analysis}

The categorical variables were expressed as percentage by using Fisher's test or chi-square test for tendency and independence.

Positional characteristics and bond status were compared using chi-square tests, t-tests, and analysis of variance (ANOVA), as applicable. Crude incidence rates and 95\% confidence intervals were calculated using time-to-event methods. The proportion of brackets remaining free of failure at any time during follow-up period was calculated using the Kaplan-Meier method. For all survival analyses, the follow-up time was defined as the period from entry into the study to the first bond failure or up to the time an individual left the study. Kaplan-Meier curves were compared using Logrank test.

A significance level of $5 \%(p<0.05)$ was adopted for all tests. The intercooled STATA software, version 9.2, was used for statistical analyses and graph construction.

\section{RESULTS}

At the end of the 6-month evaluation period, 20 brackets $(5 \%)$ out of a total of 400 had failed. Statistically significant differences $(p=0.0198)$ were observed when comparing the four adhesive systems for the number of failed brackets. The bond failure rates, risk over time, incidence rate, and confidence interval (95\%) regarding each adhesive system are described in Table 2.

Conventional Transbond XT and Transbond $X T+$ TPSEP adhesive systems were found to be statistically superior (fewer bracket failure) to Orthodontic Concise and Transbond XT without 
Table 2- Failure rates, incidence rate, and confidence interval (95\%) for each adhesive system

\begin{tabular}{ccccc}
\hline Adhesive systems & Failure rates & Incidence rate & Confidence interval & Logrank test \\
\hline $\begin{array}{c}\text { 1- Orthodontic } \\
\text { Concise }\end{array}$ & 8 & 0.00042 & $0.000168-0.0008653$ & $\mathrm{~b}$ \\
$\begin{array}{c}\text { 2- Transbond XT } \\
\text { (conventional) }\end{array}$ & 2 & 0.0001126 & $0.0000136-$ & 0.0004038 \\
3- Transbond XT & 9 & 0.0005402 & $0.000247-0.0010254$ & $\mathrm{~b}$ \\
without primer & 1 & 0.0000561 & $1.42 \mathrm{e}-06-0.0003126$ & $\mathrm{a}$ \\
4- Transbond & & & \\
XT+TPSEP & & & & \\
\hline
\end{tabular}

Same letters indicate no statistically significant difference

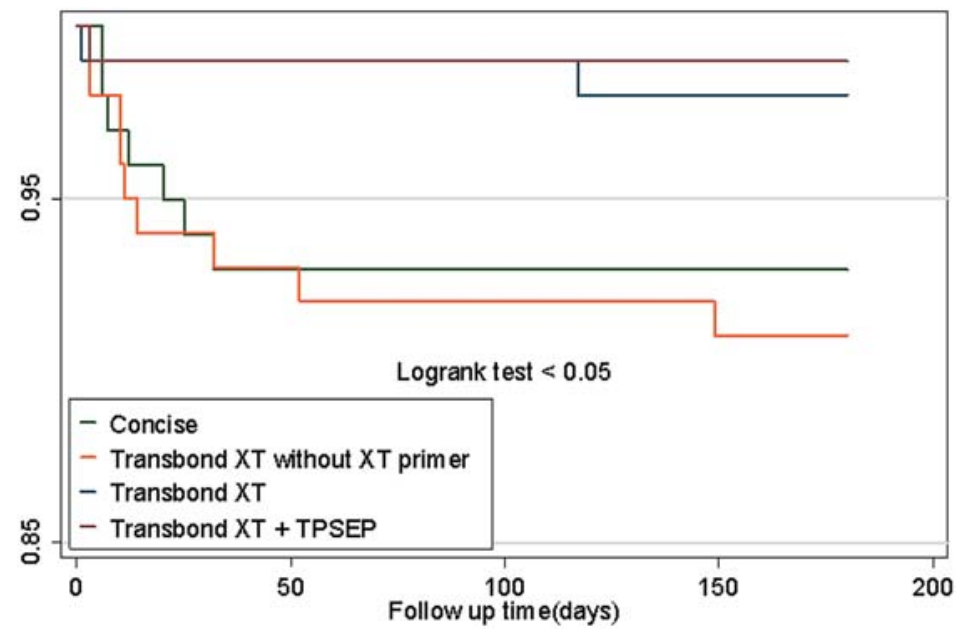

Figure 3- Kaplan-Meier survival curve for brackets bonded using the different adhesive systems

primer. On the other hand, no statistically significant differences were observed between conventional Transbond XT and Transbond XT+TPSEP $(p=0.0821)$ or between Orthodontic Concise and Transbond XT without primer $(p=0.0773)$. Figure 3 illustrates these differences according to the Kaplan-Meier survival curve.

By analyzing the number of bond failures in both dental arches, 11 failures (55\%) were recorded in the upper arch and 9 failures (45\%) in the lower arch during the same evaluation period, with no statistically significant differences $(p=0.646)$. The upper teeth affected by these bracket failures were the left and right second premolars with 5 events each, whereas the left first premolar had only 1 debonding. In the lower arch, the affected teeth were the left and right second premolars, the left central incisor, and the right lateral incisor with, respectively, 5, 2, 1, and 1 bracket failure.

By comparing the failure rate between the regions of dental arch, it was observed a statistically significant difference $(p=0.0574)$ as 2 failures $(10 \%)$ were observed in the anterior region and $18(90 \%)$ in the posterior region. The majority of the bond failure rates occurred on the left side $(n=12 ; 60 \%)$ compared with the right side $(n=8$;
$40 \%$ ), but such a difference was not statistically significant $(p=0.359)$.

Of the 400 brackets used, 100 were bonded with one of the four adhesive systems, 25 in each different quadrant for every 5 patients. No statistically significant differences were found among the quadrants and adhesive systems $(p=0.738)$. Considering all patients, 5 bond failures occurred in the upper right quadrant, 6 in the upper left quadrant, 3 in the lower right quadrant, and 6 in the lower left quadrant.

\section{DISCUSSION}

The majority of orthodontic bonding materials available for clinical use have been tested in vitro only. It is obvious that laboratory studies for assessing these materials under ideal conditions are crucial as an initial test, mainly to quantify the shear bond strength to dental enamel. However, the experimental bonding procedure differs significantly from the clinical situation, which is performed under real conditions and requires both working field and oral cavity components be controlled. This makes the bonding technique difficult, interfering with the quality of adhesion between 
the orthodontic accessories and the tooth surface. Prior to recommending a given dental material, it is crucial to test it in clinical experiments so that its characteristics and performance can be evaluated.

Studies have assessed the clinical efficacy of bonding materials $s^{4,8,25}$ by quantifying the failure rate regarding either one material ${ }^{4,10}$, two materials for two patients ${ }^{2,18}$, two materials for one patient with quadrant variations $s^{7,11,22-26}$ or even interchanging the materials between teeth ${ }^{29}$. Material variation per quadrant in the same patient was adopted to avoid that external factors, such as masticatory force, occlusive interferences, brushing style, anatomy, and type of malocclusion, interfered with the adhesive results. The present work adopted the model cited above, but with four adhesive systems (Orthodontic Concise, conventional Transbond XT, Transbond XT without primer, and Transbond XT+TPSEP) being tested in the same patient. This kind of methodological approach also allows various materials to be tested in a single experiment, thus yielding results that are more comprehensive for the same number of patients. Of the 400 bonded brackets, 20 had debonded during the 6-month period of observation, with $90 \%$ of them (18) occurring within the first two months. These results (Figure 3 ) suggest that clinical studies using shorter evaluation periods can be sufficient for demonstrating differences in the adhesive materials.

Eight failures occurred for Orthodontic Concise during the study period, which was found to be statistically inferior to that of conventional Transbond XT and Transbond XT+TPSEP systems. This inferiority can be explained by the fact that this is a self-curing material requiring a short time to be adequately handled and a long time to achieve full setting. As a result, the material is subjected to masticatory forces and occlusion. The failure rates for Orthodontic Concise found in the present study are very close to that of other works ${ }^{12,19}$ assessing this composite clinically, but in a longer period of evaluation (12 months). These results do not exclude the potential use of Orthodontic Concise in the clinical setting.

Currently, Transbond XT composite has been the most common material used as control in in vitro $^{6,9,13,14,27,28}$ and in vivo ${ }^{11,13,18,26,29}$ studies due to its good adhesiveness, easy handling, and long working time. In the present study, of the 100 brackets bonded with this material, only two failed over the 6-month evaluation period, thus confirming its high bond strength to dental enamel.

In addition to being used in the conventional manner, Transbond XT composite was also employed with two different enamel pretreatment conditions: without primer and with TPSEP. These two different applications lead to procedure simplification and decreased chair time. When Transbond XT was used without primer, 9 bracket failures were observed - a result statistically inferior to that of Transbond XT used conventionally (as indicated by the manufacturer) and Transbond XT+TPSEP, but non-statistically significant in relation to Ortodontic Concise (Table 2 and Figure 3). Despite the advantages cited above, the lack of XT primer resulted in a larger number of debonded brackets and subsequent failure in protecting the etched area not occupied by the bracket base. This means that Transbond XT system should be preferentially used according to the manufacturer's recommendations.

When enamel preparation was performed using TPSEP, only 1 bracket failure occurred. This result was statistically superior to that of both Transbond XT without primer and Orthodontic Concise, although no statistically significant difference was found in relation to conventional Transbond $\mathrm{XT}$. This is in accordance with the literature $8,11,18,24,26$ and indicates Transbond XT+TPSEP as an alternative to the conventional system. However, this result is not supported by other authors ${ }^{1,24}$ who found a larger number of bracket failures bonded with Transbond XT+TPSEP. Two studies reported the opposite ${ }^{15,29}$. Such a divergence is possibly due to methodological differences as the materials had been interchanged between patients, quadrants and teeth. Studies testing identical materials in different populations imply that culturally influenced dietary habits and sex differences can affect the failure rate of brackets in vivo ${ }^{24}$.

With regard to the failure rate, no statistically significant differences were found among the four adhesive systems with respect to the dental arches (upper and lower). Some authors ${ }^{2,11,20,23}$ have also found similar results, whereas others reported a larger number of debondings in the lower arch possibly due to occlusive forces ${ }^{18,22,24}$.

In this study, the posterior region had more bracket failures than did the anterior region. Both upper and lower premolars were the most affected teeth, totalizing $90 \%$ of bracket failures. This finding was similar to that reported by other authors ${ }^{11,18,21}$ suggesting that masticatory forces are more intense in the posterior region and the presence of a larger amount of aprismatic enamel in premolars ${ }^{30}$. Nevertheless, such a difference is not corroborated by other studies ${ }^{2,23,24}$.

The number of failures occurring on the right side $(n=8 ; 40 \%)$ compared with the left side $(n=12$; $60 \%$ ) was not found to be significantly different in the present study or elsewhere ${ }^{18,20,23}$. Few studies have assessed this variable and only one reported statistically statistical difference between the right and left sides of the dental $a^{2}$ ch $^{2}$.

Most clinical studies on the failure rate of orthodontic brackets have assessed either one 
or two adhesive systems in the same patient or materials in different quadrants. Only Murfitt, et al. ${ }^{21}$ (2006) compared failure rates between the quadrants and reported statistically significant differences. In the present work, however, four different adhesive systems were used on a quadrant-rotation basis and no significant difference was found among the quadrants of dental arch.

\section{CONCLUSIONS}

Within a 6-month evaluation period, the largest number of bracket failures occurred with Orthodontic Concise and Transbond XT without primer systems, while only few brackets failed from conventional Transbond XT and Transbond XT+TPSEP; there was no significant difference between the dental arches (upper and lower), between the dental arch sides (right and left), and among the quadrants. More bracket failures were observed in the posterior region compared with the anterior region.

\section{REFERENCES}

1- Asgari S, Salas A, English J, Powers J. Clinical evaluation of bond failure rates with a new self-etching primer. J Clin Orthod. 2002;36:687-9.

2- Banks P, Thiruvenkatachari B. Long-term clinical evaluation of bracket failure with a self-etching primer: a randomized controlled trial. J Orthod. 2007;34:243-51.

3- Bishara SE, Ajlouni R, Laffoon JF, Warren JJ. Effect of a fluoridereleasing self-etch acidic primer on the shear bond strength of orthodontic brackets. Angle Orthod. 2002;72:199-202.

4- Burgess $A M$, Sheriff $M$, Ireland AJ. Self-etching primers: Is prophylactic pumicing necessary? A randomized clinical trial. Angle Orthod. 2006;76:114-8.

5- Buyukyilmaz T, Usumez S, Karaman AI. Effect of self-etching primers on bond strength - are they reliable? Angle Orthod. 2003;73:64-70.

6- Cacciafesta V, Sfondrini MF, De Angelis M, Scribante A, Klersy C. Effect of water and saliva contamination on shear bond strength of brackets bonded with conventional, hydrophilic, and self-etching primers. Am J Orthod Dentofacial Orthop. 2003;123:633-40.

7- Cal-Neto JP, Miguel JAM. An in vivo evaluation of bond failure rates with hydrophilic and self-etching primer systems. J Clin Orthod. 2005;39:701-2.

8- Cal-Neto JP, Miguel JA, Zanella E. Effect of self-etching primer on shear bond strength of adhesive precoated brackets in vivo. Angle Orthod. 2006;76:127-31.

9- Dorminey JC, Dunn WJ, Taloumis LJ. Shear bond strength of orthodontics brackets bonded with a modified 1-step etchant-andprimer technique. Am J Orthod Dentofacial Orthop. 2003;124:4103.

10- Duan Y, Chen X, Wu J. Clinical comparison of bond failures using different enamel preparations of severely fluorotic teeth. J Clin Orthod. 2006;40:152-4.

11- Elekdag-Turk S, Isci D, Turk T, Cakmak F. Six-month bracket failure rate evaluation of a self-etching primer. Eur J Orthod. 2008;30:211-6
12- Fowler PV. A twelve-month clinical trial comparing the bracket failure rates of light-cured resin-modified glass-ionomer adhesive and acid-etched chemical-cured composite. Aust Orthod J. 1998;15:186-90.

13- Grandhi K, Combe EC, Speidel TM. Shear bond strength of stainless steel orthodontic brackets with a moisture-insensitive primer. Am J Orthod Dentofacial Orthop. 2001;119:251-5.

14- Grubisa HS, Heo G, Raboud D, Glover KE, Major PW. An evaluation and comparison of orthodontic bracket bond strengths achieved with self-etching primer. Am J Orthod Dentofacial Orthop. 2004;126:213-9.

15- Ireland $A L$, Knight $H$, Sherriff $M$. An in vivo investigation into bond failure rates with a new self-etching system. Am J Orthod Dentofacial Orthop. 2003;124:323-6.

16- Jassem HA, Retief DH, Jamison HC. Tensile and shear strengths of bonded and rebonded orthodontic attachments. Am J Orthod. 1981;79:661-8.

17- Lill DJ, Lindauer SJ, Tüfekçi E, Shroff B. Importance of pumice prophylaxis for bonding with self-etch primer. Am J Orthod Dentofacial Orthop. 2008;133:423-6.

18- Manning N, Chadwick SM, Plunkett D, Macfarlane TV. A randomized clinical trial comparing "one step" and "two step" orthodontic bonding systems. J Orthod. 2006;33:276-83.

19- Miguel JA, Almeida MA, Chevitarese O. Clinical comparison between a glass ionomer cement and a composite for direct bonding of orthodontic brackets. Am J Orthod Dentofacial Orthop. 1995;107:484-7.

20- Moin K. Indirect bonding of orthodontic attachments. Am J Orthod. 1977; 72:261-75.

21- Murfitt PG, Quick AN, Swain MV, Herbison GP. A randomized clinical trial to investigate bond failure rates using a self-etching primer. Eur J Orthod. 2006;28:444-9.

22- Noble RR, Salas-Lopez A, English JD, Powers JM. Clinical evaluation of orthodontic self-etching primers. Texas Dent $\mathrm{J}$. 2006;123:274-8.

23- Pandis $\mathrm{N}$, Eliades T. A comparative in vivo assessment of the long-term failure rate of 2 self-etching primers. Am J Orthod Dentofacial Orthop. 2005;128:96-8.

24- Pandis N, Polychronopoulou A, Eliades T. Failure rate of selfligating and edgewise brackets bonded with conventional acid etching and a self-etching primer: a prospective in vivo study. Angle Orthod. 2006;76:119-22.

25- Pasquale A, Weinstein M, Borislow AJ, Braitman LE. In-vivo prospective comparison of bond failure rates of 2 self-etching primer/adhesive systems. Am J Orthod Dentofacial Orthop. 2007; 132:671-4.

26- Reis A, Santos JE, Loguercio AD, Bauer JRO. Eighteen-month bracket survival rate: conventional versus self-etch adhesive. Eur J Orthod. 2008;30:94-9.

27- Romano FL, Correr-Sobrinho L, Magnani MBBA, Nouer DF, Sinhoretti MAC, Correr AB. Shear bond strength of metallic brackets bonded under various enamel conditions. Braz Oral Res. 2006;20(Spec Iss):28-33.

28- Romano FL, Tavares SW, Nouer DF, Consani S, Magnani MBBA. Shear bond strength of metallic orthodontic brackets bonded to enamel prepared with self-etching primer. Angle Orthod. 2005;75:849-53.

29- Santos JE, Quioca J, Loguercio AD, Reis A. Six-month bracket survival with a self-etch adhesive. Angle Orthod. 2006;76:863-8. 30- Whittaker DK. Structural variations in the surface zone of human tooth enamel observed by scanning electron microscopy. Arch Oral Biol. 1982;27:383-92. 